\title{
HPV-Independent Gastric Type Adenocarcinoma of the Uterine Cervix presenting as Ovarian Masses: A Case Report and Review of Literature*
}

\author{
Joseph Antoine Chatto ${ }^{1}$ and Annette Salillas ${ }^{1,2}$
}

${ }^{1}$ Department of Pathology, Governor Celestino Gallares Memorial Hospital, Tagbilaran City, Bohol, Philippines

${ }^{2}$ Matias H. Aznar Memorial College of Medicine, Cebu City, Philippines

\section{ABSTRACT}

Adenocarcinoma, HPV-independent, gastric type of the uterine cervix comprises only $10-15 \%$ of all cervical adenocarcinomas. A rare case of which, with metastasis to the uterine corpus and bilateral ovaries, is described. A 43-year-old female (GOPO) presented with menorrhagia and right flank pain radiating to the hypogastrium. Physical examination revealed an immovable, tender mass at the right lower quadrant with a nodular, firm cervix. Transabdominal ultrasound revealed multiseptated ovarian masses. The right and left ovaries were sent for frozen section and was diagnosed as Mucinous Cystadenoma and Mature Cystic Teratoma, respectively. Hysterectomy revealed a detached and fragmented cervix with irregular, abnormally shaped glands lined by a single layer of columnar cells with bland, basally located nuclei and clear cytoplasm associated with desmoplasia, findings which were also seen in the endomyometrium and ovaries. These tumor cells were CK7 positive and negative for ER, PR, CK20 and CDX2. The patient died six months after surgery. The presence of benign appearing glands is a diagnostic challenge. Despite the appearance, they may be malignant and should be investigated rigorously.

Key words: cervical adenocarcinoma, gastric-type cervical adenocarcinoma, hpv-independent cervical adenocarcinoma

ISSN 2507-8364 (Online)

Printed in the Philippines.

Copyright $₫ 2021$ by the PJP.

Received: 18 March 2021

Accepted: 5 May 2021.

Published online first: 30 June 2021

https://doi.org/10.21141/PJP.2021.06

Corresponding author: Joseph Antoine F. Chatto, MD, DPSP E-mail: antoinechatto@gmail.com

* This case report was presented as poster presentation at the XXXII International Academy of Pathology Congress in Dead Sea, Jordan on October 14-19, 2018.

\section{INTRODUCTION}

Adenocarcinoma, HPV-independent, Gastric Type, initially called adenoma malignum, was first described by Gusserow in $1870 .{ }^{1}$ It was named as such due to its resemblance to endocervical glands without malignant features. ${ }^{2}$ In 1975 , Silverberg proposed a designation of minimal deviation adenocarcinoma (MDA) to accurately represent its clinical behavior. $^{3}$ In 2014, the WHO classification grouped extremely well-differentiated mucinous adenocarcinoma with gastric type differentiation including Minimal Deviation Adenocarcinoma as Mucinous Carcinoma, Gastric Type. ${ }^{4}$ The WHO Classification of Tumors for Female Genital Tumors updated the nomenclature to Adenocarcinoma, HPV-independent, gastric type in 2020, setting it apart from the other mucinous adenocarcinomas of the uterine cervix, which are HPV-dependent. ${ }^{5}$ This variant of mucinous adenocarcinoma is considered to be a separate entity because it has distinct features and clinical course.$^{6}$ It is rare, accounting for only $10-15 \%$ of all cervical adenocarcinomas and is often misdiagnosed. ${ }^{2,5,7-11}$ Although the pattern is benign, this type of tumor is highly malignant and has poor prognosis. ${ }^{10}$ In this report, we describe a case of mucinous carcinoma with gastric differentiation metastasizing to the uterine corpus and bilateral ovaries and compare it with previous reports.

A 43-year-old female (G0P0) presented with a ten-month history of right flank pain radiating to the hypogastric area and a 6-month history of prolonged menstrual bleeding lasting ten to eleven days. On physical examination, the abdomen was flat and soft with a $5.0 \times 5.0 \mathrm{~cm}$ fixed and tender palpable mass at the right lower quadrant. 
The uterine cervix was closed, nodular and firm. Transabdominal ultrasound revealed an abdominopelvic septated, cystic mass, ovarian in origin measuring $11.9 \mathrm{x}$ $9.1 \times 7.5 \mathrm{~cm}$. Total abdominal hysterectomy with bilateral salpingo-oophorectomy, appendectomy and frozen section were performed at our institution. The cervix was fragile and was damaged during surgery. On frozen section, a diagnosis of Mucinous Cystadenoma (right ovary) and Mature Cystic Teratoma (left ovary) was given. The fixed specimens were then processed for final diagnosis.

\section{CASE}

The uterus was previously opened, tan gray, rubbery measuring $9.0 \times 7.0 \times 6.0 \mathrm{~cm}$. The uterine cavity was 4.0 $\mathrm{cm}$ in depth. Endometrial thickness measured $0.1 \mathrm{~cm}$ while the myometrium measured $4.0 \mathrm{~cm}$ with slit-like spaces (Figure 1A). The detached cervix was fragmented, tan gray and firm measuring $3.0 \times 1.5 \mathrm{~cm}$ (Figure 1B). The right ovary was tan cream, smooth and rubbery measuring $17.0 \times 13.5 \times 9.0 \mathrm{~cm}$. The cut surface was multiloculated with mucinous fluid. No solid areas were noted (Figure 1C). The fallopian tube was tan gray, smooth and rubbery measuring $6.0 \times 0.9 \mathrm{~cm}$. Cut section shows an empty lumen. The left ovary was tan cream to gray, smooth and soft in consistency measuring $12.0 \times 8.5 \times 7.0 \mathrm{~cm}$. Cut section showed a biloculated mass, one measured $7.0 \mathrm{~cm}$ in diameter containing hair strands and sebum. The smaller cyst measuring $3.0 \mathrm{~cm}$ contained mucinous fluid (Figure 1D). Attached to the left ovary was a gray tan, fallopian tube measuring $4.0 \times 0.9 \mathrm{~cm}$, which was grossly unremarkable. The appendix appeared tan gray with smooth surface measuring $5.0 \times 1.0 \mathrm{~cm}$ with brown materials seen on cut section.

Microscopically, the cervix was filled with widely spaced irregular and angulated glands infiltrating deep into the cervical stroma. They are lined by a single layer of columnar cells with bland, basally located nuclei and clear cytoplasm associated with desmoplasia. Some of them formed leaf-like patterns while others were cystically dilated (Figure 2A). Neoplastic glands were also seen in the endometrium and myometrium, majority of which were in the myometrium encroaching from the cervix (Figure 2B), as well as in both ovaries (Figures 2C and 2D). Sections taken from the right ovary showed glands in close proximity to thick-walled blood vessels (Figure 2C). The left ovary had areas showing hair follicles, skin, cartilage, smooth muscles, and sebaceous glands. Thick and thin-walled congested vessels interspersed with few mature adipose tissues were noted as well. The appendix showed proliferating lymphoid nodules with active germinal centers.

On immunohistochemical staining, the tumor cells in the cervix, endomyometrium and ovaries were positive for CK7 and negative for ER, PR, CK20 and CDX2.

Based on the above morphological and immunohistochemical findings, a final diagnosis of Mucinous Carcinoma, Gastric Type was made. The patient died six months after surgery.
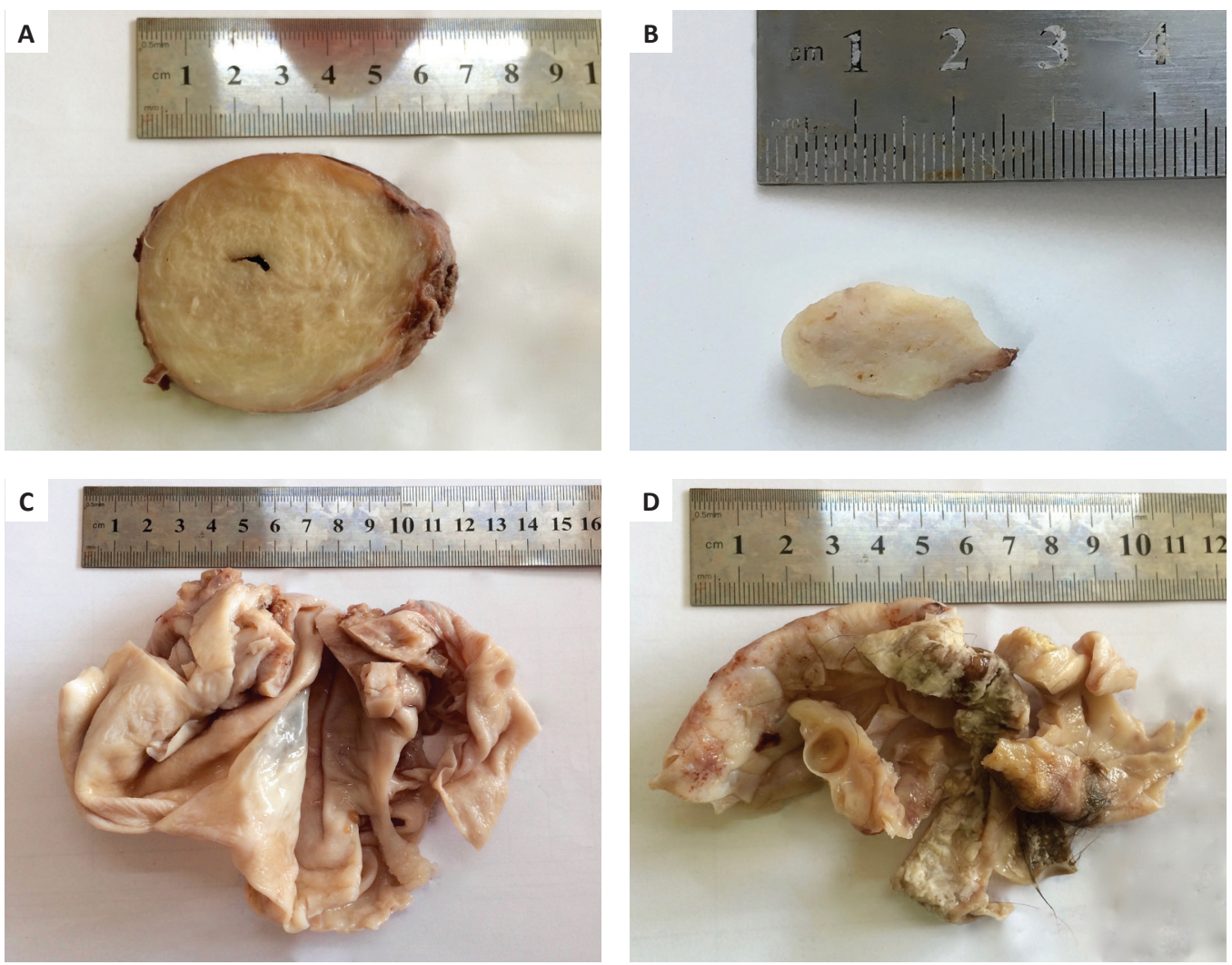

Figure 1. Gross examination: (A) Cross section of the uterus showing the endometrium and myometrium; (B) A fragment of the cervix, which was damaged during surgery; (C) Right ovary (opened) showing a multiloculated cyst; (D) Left ovary (opened) filled with hair strands and sebaceous material. 

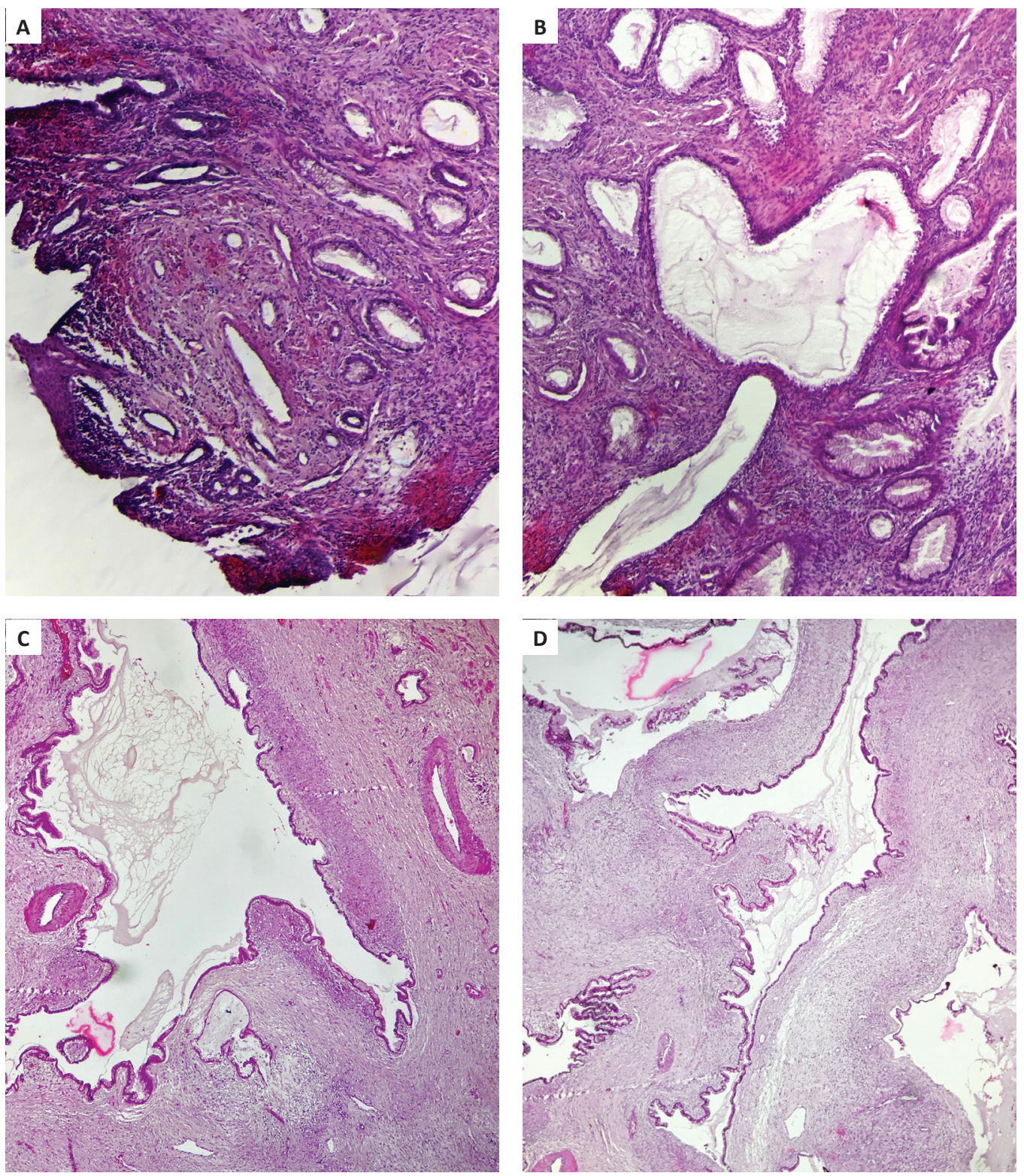

Figure 2. Histopathologic Features on H\&E: (A) Cervix with widely spaced glands lined by a single layer of columnar cells. The cells have bland, basally located nuclei and clear cytoplasm surrounded by moderate desmoplasia, 4x; (B) Endomyometrium with similar neoplastic mucinous glands, 4x; (C) Benign appearing glands in the right ovary near thick-walled blood vessels, 4x; (D) Neoplastic glands in the left ovary with similar morphologic features as those seen in the cervix, $4 \mathrm{x}$.

\section{DISCUSSION}

Adenocarcinoma, HPV-independent, gastric type of the uterine cervix accounts for only $10-15 \%$ of all cervical adenocarcinomas with the average age at diagnosis of 45 years. ${ }^{2,5,7-11}$ Clinical symptoms may be abnormal vaginal discharge and uterine bleeding as well as an enlargement of the cervix associated with erosion and hardening., ${ }^{2,12}$ Early physical examinations may not reveal any specific findings. At later stages, however, cervical hypertrophy with granular appearance of the cervical os may be present. ${ }^{10}$ Due to the late diagnosis, metastasis to the ovaries may already be seen at presentation, ${ }^{4}$ which is similar to our case. The patient presented with heavy menstrual bleeding lasting for ten to eleven days. The cervix was also nodular and firm on internal examination.
Despite the deep infiltration of this type of carcinoma, a visible lesion is not seen in many cases, ${ }^{7}$ which is also comparable to this case. Radiologic findings are not specific. Ultrasonography, CT and MRI may reveal intrauterine and/or vaginal fluid accumulation. ${ }^{10}$ Moreover, multilocular lesions with solid components from the endocervical glands to the deep cervical stroma may be seen on MRI.1,13 On contrast imaging using bolus intravenous gadolinium-diethylene triaminepentaacetic acid (Gd-DTPA), rough, irregular cyst walls with fine granular appearance may be detected. ${ }^{10}$ A recent study by Mills using PET combined with CT showed that these lesions may be intensely hypermetabolic. ${ }^{14}$

Cytological evaluation and biopsies have low detection rates, which could delay accurate diagnosis, eventually 
leading to poor prognosis. ${ }^{1}$ As such, deep conization or hysterectomy may be necessary to make a proper diagnosis. $^{2}$ Kojima et al., established the criteria for histologic diagnosis wherein tumor cells must have clear or pale, voluminous, eosinophilic cytoplasm and distinct cell borders. ${ }^{6}$ However, there is still a lack of criteria to differentiate benign hyperplastic lesions, MDA and common adenocarcinoma. There are also interobserver differences in the interpretations of cellular atypia and invasion. ${ }^{15}$

This neoplasm is a well-differentiated form in the spectrum of gastric type mucinous adenocarcinoma. ${ }^{6} \mathrm{On}$ low power, the glands have different sizes with irregular and distorted forms and angular projections. Complex outlines and desmoplasia are usually present. On higher magnification, the nuclei are basally located and bland without prominent nucleoli. ${ }^{7}$ Some areas may show cribriform pattern, solid areas and infolded papillae. ${ }^{6}$ Due to its benign histologic appearance, frequent diagnostic difficulties are encountered, especially in small biopsy specimens. ${ }^{2}$ The most reliable criteria for assessing its malignant nature are the haphazard arrangement of the irregular and angulated glands extending beyond the level of the normal endocervical glands as well as the presence of occasional mitosis. The stromal reaction is a helpful indicator of its infiltrative nature. ${ }^{7}$ Some of the major differential diagnoses are deeply positioned nabothian cysts, tunnel clusters, microglandular hyperplasia and mesonephric hyperplasia. ${ }^{2}$

In 2018, the International Endocervical Adenocarcinoma Criteria and Classification (IECC) published a new pathogenetic classification for invasive endocervical adenocarcinomas, wherein the tumors were classified as HPV-associated adenocarcinoma (HPVA) and no or limited HPVA features (NHPVA). Tumors with apical mitosis and/or apoptotic bodies were classified as HPVA while tumors without these features were classified as NHPVA. These groups were then subclassified using existing histomorphologic criteria and supported by p16 immunophenotype and HPV status. The study showed that mucinous carcinomas comprise a mixture of HPVA and NHPVA types and the gastric type was the major NHPVA type.$^{16}$ Compared to HPVA, NHPVAs were larger, occurred in older patients, presented at a higher stage and has different responses to standard therapy. ${ }^{17}$

In our case, immunohistochemical staining of the tumor cells in the cervix and endomyometrium were $\mathrm{CK} 7$ positive and negative for ER, PR, CK20 and CDX2. Both ovaries were also positive for CK7 and negative for ER, PR, CK20 and CDX2. Given their negative staining for ER and $\mathrm{PR}$, a carcinoma of endometrial origin is not considered since tumor cells from the endometrium are expected to be positive. The negative staining for CK20 supports an endocervical primary. Furthermore, the possibility of an intestinal primary tumor is not considered because the tumor cells are negative for CK20 and CDX2. Gastric type adenocarcinoma does not express the characteristic Mullerian-type substances such as ER, PR and CA125 and a proportion of cells contain gastric epithelial substances such as gastric mucin and CEA. ${ }^{7}$ Tumor cells also show neutral mucin production and positive staining for
HIK1083 and MUC6 suggesting a gastric phenotype. ${ }^{6}$ HIK 1083 is an antibody directed against pyloric gland mucin and is negative in most cervical adenocarcinomas and endocervical glandular lesions. ${ }^{18} \mathrm{P} 16$ is usually negative or only focally positive, in contrast to HPVassociated adenocarcinomas where diffuse positive staining is seen. ${ }^{19}$ Mutations in the STK11 are also observed in this type of adenocarcinoma making it commonly associated with Peutz-Jeghers syndrome. ${ }^{20}$ This mutation has been noted in about half of this neoplasm and is more likely to lose expression of mullerian-type markers. ${ }^{4,21}$ A clonal assay study by Li Gong et al., demonstrated that this tumor type was monoclonal, which makes it a true neoplastic lesion, whereas adenomatous hyperplasia and normal cervical tissues were polyclonal. ${ }^{22}$ A clonality analysis also demonstrates an $\mathrm{X}$ chromosome inactivation in MDA. ${ }^{23}$

Surgical treatment is the most successful option. However, there is still no standard surgical treatment or adjuvant therapy established. ${ }^{1}$ The use of neoadjuvant chemotherapy and radiotherapy has become an alternative approach and the response to this therapy before surgery can predict the prognosis. ${ }^{6}$ Some authors have described purely surgical management while others have described the use of adjuvant therapy with chemotherapy or external beam radiation. ${ }^{13}$

Gastric type adenocarcinoma of the uterine cervix is reported to have an aggressive clinical course. The five-year survival rate of patients with gastric type adenocarcinoma $(38 \%)$ is substantially lower than that of patients with the usual type of uterine cervical adenocarcinoma (74\%). Moreover, a study by Kojima, et al. indicates that the 2-year survival rate of patients with any stage of minimal deviation adenocarcinoma is $20-30 \%$, whereas that of patient with stage I cervical adenocarcinoma is around 50\%. ${ }^{6}$ Early peritoneal dissemination and distant metastasis is usually observed as this tumor has a propensity to spread to the ovaries, abdominal wall or peritoneum, omentum and urinary tract. ${ }^{24,25}$ Similar findings were also seen by Nishio et al., in a multi-institutional study wherein this tumor is associated with histopathological predictors of poor outcomes, such as bulky mass, deep stromal invasion, lymphovascular space invasion, parametrial invasion, ovarian metastasis and positive ascitic fluid cytology. ${ }^{26}$ In our case, the tumor has spread to the bilateral ovaries. Peritoneal invasion and distant metastasis were not noted. In contrast to the abovementioned statistics, some studies have reported conflicting results stating that survival rates and distribution of metastases are only similar to those of a classic well-differentiated adenocarcinoma. ${ }^{7}$

\section{CONCLUSION}

Adenocarcinoma, HPV-independent, gastric type, of the uterine cervix is a rare neoplasm which poses a diagnostic challenge because of its deceptively benign appearance. It is reported to be highly aggressive, prone to metastasize and has poor prognosis. Symptoms, physical examination, and radiologic evaluation are nonspecific. Also, cytological evaluation may not be useful and conization or hysterectomy is necessary to assess the invasion, which is one of the diagnostic criteria. The presence of bilateral ovarian masses associated with a cervical pathology 
should be investigated rigorously because there is a high chance of malignancy and metastasis despite the benign appearance. Because it is often misdiagnosed, it is usually in its advanced stage at the time of diagnosis.

\section{ETHICAL CONSIDERATION}

An Ethics Approval was obtained from the institution. The hospital assumes full responsibility of the case in accordance with the Data Privacy Act of the Philippines under Republic Act Number 10173. The authors ensured that the case report has no identifying information and has been sufficiently anonymized.

\section{STATEMENT OF AUTHORSHIP}

Both authors certified fulfillment of ICMJE authorship criteria.

\section{AUTHOR DISCLOSURE}

Both authors declared no conflict of interest.

\section{FUNDING SOURCE}

None.

\section{REFERENCES}

1. Nishii Y, Fukuda T, Imai K, et al. Minimal deviation mucinous adenocarcinoma of the uterine cervix that proved difficult to differentiate from endometrial cancer: A case report. Oncol Lett. 2014;8(6):2481-4. PMID: 25364411. PMCID: PMC4214510. https://oi. org/10.3892/ol.2014.2532.

2. Kushwaha R, Yadav YK. A rare case of minimal deviation mucinous adenocarcinoma of the uterine cervix and review of literature. Clin Cancer Inves J. 2013;2(4):362-4. https;//doi.org/ 10.4103/22780513.121549 .

3. Silverberg SG, Hurt WG. Minimal deviation adenocarcinoma ("adenoma malignum") of the cervix: a reappraisal. Am J Obstet Gynecol. 1975;121(7): 971-5. PMID: 1115185 . https://doi.org/10.1016/00029378(75)90920-5.

4. Wilbur DC, Colgan TJ, Ferenczy AS, et al. Glandular tumours and precursors. In: Kurman RJ, Carcangiu ML, Herrington CS, Young RH, eds. WHO classification of female reproductive organs, 4th ed. Lyon: IARC; 2014.

5. Park KJ, Kong CS, Ohishi Y, Parra-Herran C. Adenocarcinoma, HPV-independent, gastric type, of the uterine cervix. In: Herrington CS, ed. WHO classification of tumours female genital tumours 5th ed. Lyon: IARC; 2020; 374-5.

6. Zhang Y, Liang L, Euscher ED, Liu J, Ramalingam P. Gastric-type mucinous adenocarcinoma of the uterine cervix with neoadjuvant therapy mimicking clear cell carcinoma. Int J Clin Exp Pathol. 2015;8(9):11798803. PMID: 26617929. PMCID: PMC4637745.

7. Chang E, Lee E, Kim K, et al. Minimal deviation adenocarcinoma, mucinous type, of the uterine cervix - report of a case with extensive metastasis to the uterine corpus and bilateral adnexae. Korean J Pathol. 2004;38:121-5. http://www.koreanjpathol.org/ upload/journal/2004/2004_0121.pdf.

8. Mutter G, Prat J. Pathology of the female genital tract, 3rd ed. London: Churchill Livingstone Elsevier ;2014.

9. Fletcher C. Diagnostic histopathology of tumors, 4th ed, vol. 1. Philadelphia: Elsevier; 2013.

10. Jie C, Shen Z, Hui Z, Jin-Xiao L, Zhong-Qiu. Clinical analysis of minimal deviation adenocarcinoma of the cervix: a report of five cases. Ai Zheng. 2008;27(12):1310-4. PMID: 19080000.

11. Villegas-Cruz C, Arias AL, Montiel DP, ChanonaVilchis J, Montalvo LAH, de Leon DC. Minimal deviation adenocarcinoma associated with high grade squamous cervical intraepithelial neoplasia. Obstet Gynecol Cases Rev. 2015;2:4. https://clinmedjournals. org/articles/ogcr/ogcr-2-047.pdf.

12. Kwon KW, Chin S, Kim HK, Koh ES, Kim KR, Nam KH. Minimal deviation endometrioid adenocarcinoma of the uterine cervix: a case report. Kor J Pathol. 2008;42:240-3. http://www.koreanjpathol.org/upload/ journal/2008/2008_0240.pdf.

13. Senar AC, Paño B, Saco A, Nicolau C. Magnetic resonance imaging of adenoma malignum of the uterine cervix with pathologic correlation: a case report. Radiol Case Rep. 2016;(11):323-7. PMID: 27920853. PMCID: PMC5128363. https://doi.org/ 10.1016/j.radcr.2016.08.008.

14. Mills K, Shuen P, Zolis L. Adenoma malignum presenting with profound yyponatremia. J Obstet Gynaecol Can. 2015;37(7):624-7. PMID: 26366819. https://doi.org/10.1016/S1701-2163(15)30200-0.

15. Tsuda H, Mikami Y, Kaku T, et al. Interobserver variation in the diagnosis of adenoma malignum (minimal deviation adenocarcinoma) of the uterine cervix. Pathol Int. 2003;53(7):440-9. PMID: 12828609. https://doi.org/10.1046/j.1440-1827.2003.01497.x.

16. Stolnicu S, Barsan I, Hoang L, et al. International Endocervical Adenocarcinoma Criteria and Classification (IECC): a new pathogenetic classification for invasive adenocarcinoma of the endocervix. Am J Surg Pathol. 2018;42(2):214-26. PMID: 29135516. PMCID: PMC5762258. https://doi.org/10.1097/PAS. 0000000000000986.

17. Park KJ. Cervical adenocarcinoma: integration of HPV status, pattern of invasion, morphology and molecular markers into classification. Histopathology 2020, 76(1):112-7. PMID: 31846527. https://doi.org/ 10.1111/his.13995.

18. McLuggage, WG. Immunohistochemistry in the differential diagnosis of female genital tract pathology. In: Nucci M, et al. Gynecologic pathology: a volume in the series: foundations of diagnostic pathology. Elsevier Churchill Livingstone; 2009.

19. Moat M, O'Donnell RL, McCluggage WG, Ralte A, Edmondson RJ. Gastric-type adenocarcinoma of the cervix in a patient with Lynch syndrome: a case report. Gynecol Oncol Rep. 2014;10:41-3. PMID: 26082936. PMCID: PMC4458744. https://doi.org/10.1016/j. gynor.2014.03.002.

20. Kusanagi Y, Kojima A, Mikami Y, et al. Absence of high-risk human papillomavirus (hpv) detection in endocervical adenocarcinoma with gastric morphology 
and phenotype. Am J Pathol. 2010;177(5):2169-75. PMID: 20829441. PMCID: PMC2966776. https://doi. org/10.2353/ajpath.2010.100323.

21. Kuragaki C, Enomoto T, Ueno Y. Mutations in the STK11 Gene Characterize Minimal Deviation Adenocarcinoma of the Uterine Cervix. Laboratory Investigation. 2003;83(1);35-45. PMID: 20416098. PMCID: PMC2877003. https://doi.org/10.1186/17461596-5-25.

22. Gong L, Zhang WD, Liu XY, et al. Clonal status and clinicopathological observation of cervical minimal deviation adenocarcinoma. Diagn Pathol. 2010;5:25. PMID: 20416098. PMCID: PMC2877003. https://doi. org/10.1186/1746-1596-5-25.

23. He M, Jackson C, Gubrod R, et al. KRAS mutations in mucinous lesions of the uterus. Am J Clin Pathol. 2015;143(6):778-4. PMID: 25972319. https://doi. org/10.1309/AJCP69RBNUHHOJRI.
24. Banerjee P, Mitra PS, Bhowmik P, Soreng PS, Mukherjee B, Choudhury S. Minimal deviation adenocarcinoma ("adenoma malignum") of the cervix: a diagnostic dilemma. IOSR J Dental Med Sciences. 2013;9(4):12-4. http://www.iosrjournals.org/iosr-jdms/ papers/Vol9-issue4/C0941214.pdf.

25. Lin M, Kim K, Ro J. Gastric-type endocervical adenocarcinoma: review of clinicopathologic characteristics and recent advances. J Gynecol Res Obstet. 2020;6(3):72-5. https://doi.org/10.17352/jgro.000091.

26. Nishio S, Mikami Y, Tokunaga H, et al. Analysis of gastric-type mucinous carcinoma of the uterine cervixAn aggressive tumor with a poor prognosis: A multiinstitutional study. Gynecol Oncology. 2019;153(1): 13-9. PMID: 30709650. https://doi.org/10.1016/j. ygyno.2019.01.022.

Disclaimer: This journal is OPEN ACCESS, providing immediate access to its content on the principle that making research freely available to the public supports a greater global exchange of knowledge. As a requirement for submission to the PJP, all authors have accomplished an AUTHOR FORM, which declares that the ICMJE criteria for authorship have been met by each author listed, that the article represents original material, has not been published, accepted for publication in other journals, or concurrently submitted to other journals, and that all funding and conflicts of interest have been declared. Consent forms have been secured for the publication of information about patients or cases; otherwise, authors have declared that all means have been exhausted for securing consent.

\section{Publish in the new PJP.} Visit our website: http://philippinejournalofpathology.org 